\title{
Impact of physical therapy on muscle strength in patients with degenerative hip changes
}

\author{
Edina Tanović ${ }^{1}$ Adnana Talić-Tanović2 , Đemil Omerović², Mevludin Mekić3, \\ Vanda Zovko-Omeragićc ${ }^{\text {, Senad Selimović }}{ }^{5}$
}

\begin{abstract}
${ }^{1}$ Department for Physical Medicine and Rehabilitation, Clinical Center of the University of Sarajevo, Sarajevo, Bosnia and Herzegovina, ${ }^{2}$ Department of Orthopedics and Traumatology, Clinical Center of the University of Sarajevo, Sarajevo, Bosnia and Herzegovina, ${ }^{3}$ Department for Heart Diseases, Blood Vessels and Rheumatology, Clinical Center of the University of Sarajevo, Sarajevo, Bosnia and Herzegovina, ${ }^{4}$ Faculty of Health Studies, University of Mostar, Mostar, Bosnia and Herzegovina, 5JZU BRC Aquaterm, Olovo, Bosnia and Herzegovina
\end{abstract}

\begin{abstract}
Introduction: An increasing number of people suffer from degenerative diseases of the hip joints. Many studies report the long-term outcomes of invasive therapy in the treatment of coxarthrosis. However, results of prolonged conservative treatment are scarce. The purpose of this study was to evaluate the effects of prolonged conservative physical therapy on functional recovery of degenerative hip changes.

Methods: We retrospectively analyzed 47 patients diagnosed with coxarthrosis according to the American College of Rheumatology criteria. Data on sex and age, duration of physical therapy, the type of physical therapy, the values of manual muscle test (MMT) at admission and discharge, were taken from the medical records of the patients.
\end{abstract}

Results: There were $76.6 \%$ female and $23.4 \%$ male patients. Half of the patients were 70 or more years old. Majority of patients were treated by kinesiotherapy $(74.5 \%)$ and had significantly different $(p<0.05)$ values of MMT at admission and discharge. Duration of treatment was $10-20$ days for $23.4 \%$ of patients, while $29.8 \%$ received treatment for 31-40 days.

Conclusion: According to our results, MMT significantly improved at the end of the treatment. Thus, physical therapy had a positive effect on the recovery of muscle strength in patients with coxarthrosis.

Keywords: Physical therapy; manual muscle test (MMT); hip degeneration

\section{INTRODUCTION}

Osteoarthritis (OA) is a chronic, progressive, and primary degenerative disease of the synovial joints

\footnotetext{
*Corresponding author: Edina Tanović, Department for Physical Medicine and Rehabilitation, Clinical Center of the University of Sarajevo, Bolnička 25, 71000 Sarajevo, Bosnia and Herzegovina Email: tanovicedina@hotmail.com
}

Submitted: 15 January 2018/Accepted: 13 March 2018

DOI: https://doi.org/10.17532/jhsci.2018.491 which is characterized by focal loss of articular cartilage and reactive phenomena in the subchondral bone, synovium, and the periarticular structures (1). OA probably begins with localized, uneven degradation of articular cartilage (2). This process is accompanied with consequent thinning of the subchondral bone, bone reproducing the periphery of the hinge (formation of osteophytes) as well as moderate to medium synovial inflammation. Initial events leading to the onset of OA have not been fully 
established, but are likely to occur due to abnormal signals that alter the chondrocyte phenotype, with the consequent synthesis of proteins that degrade the matrix and cause joint destruction (3).

Degenerative osteoarthritis is one of the four most common rheumatic diseases. Modern lifestyle, above all low physical activity, common obesity and aging of the population, increase the number of patients with coxarthrosis in everyday practice (4). OA, including coxarthrosis, may be primary (idiopathic) or secondary. The cause of primary $\mathrm{OA}$ after the elimination of anatomic abnormalities and other specific causal entities remains unknown. However, there are numerous causes of secondary OA, such as congenital deformities, trauma, sepsis, systemic metabolic or endocrine disorders. Coxarthrosis is manifested by progressive pain at rest, especially in the movements. Also, it may bring about reduced amplitudes leading to impaired mobility, hindered or denied carrying out of everyday activities, resulting in a decline in quality of life (4). In this study, we aimed to evaluate the effects of different modalities of physical therapy on the recovery of muscle strength in degenerative hip changes.

\section{METHODS}

\section{Patients}

We conducted a retrospective study at the Department of Physical Medicine and Rehabilitation at the Clinical Center of the University of Sarajevo. A total of 47 patients (11 men and 36 women) diagnosed with coxarthrosis according to the American College of Rheumatology criteria (5). We excluded patients with hip dysplasia, inflammatory disease, obesity, trauma, fracture of the hip. Data on sex and age, duration of the physical therapy, type of the physical therapy, and the values of manual muscle test (MMT) at admission and discharge, were taken from the medical records of the patient.

\section{Statistical analysis}

We used descriptive statistics to process the data. Microsoft Excel was used to calculate the arithmetic mean and percentages. We used two-tailed t test to test the differences between the groups.

\section{RESULTS}

We analyzed the data of 47 patients hospitalized and treated at the Department for Physical Medicine and Rehabilitation at the Clinical Center of the University of Sarajevo.

Out of the total number of patients, 23.4\% (11) were males, and $76.6 \%$ (36) were females.

The average age of respondents was 67.53 years (range 37-86 years). Magnetotherapy was applied in 17 patients $(36.2 \%)$, ultrasound in 8 patients $(17 \%)$, transcutaneous electrical nerve stimulation (TENS) in 20 patients (42.6\%), and manual massage in 7 patients $(14.9 \%)$. In 6 patients $(12.8 \%)$ hydrocollator was used, kinesiotherapy was used in 35 patients (74.5\%), 24 patients $(51.1 \%)$ were treated with interferent current, and 12 patients (25.5\%) received diadynamic current therapy. Also, electrophoresis was performed on 4 patients $(8.5 \%)$, galvanization was applied in 5 patients (10.6\%), sonophoresis was used in 1 patient $(2.1 \%)$, and cryomassage in 3 patients $(6.4 \%)$ (Table 1$)$.

Eleven patients $(23.4 \%)$ were treated from 10 to 20 days, while 14 (29.8\%) patients were treated for 31 to 40 days.

The most frequent MMT score was 3, observed in 14 patients $(29.8 \%)$, while the least frequent were 1 and $4(2.1 \%)$. On admission, the lowest score was 1 while the highest was 4 . On discharge, the most frequent MMT score was 3 (14 patients, 29.8\%, while the least frequent was $2+$ (1 patient, $2.1 \%)$. Also,

TABLE 1. The applied physical therapy in the treatment

\begin{tabular}{lcc}
\hline Type of therapy & $\begin{array}{c}\text { Applied - number } \\
\text { of patients (\%) }\end{array}$ & $\begin{array}{c}\text { Not applied- Number } \\
\text { of patients (\%) }\end{array}$ \\
\hline Magnet therapy & $17(36.2)$ & $30(63.8)$ \\
Ultrasound & $8(17)$ & $39(83)$ \\
TENS & $20(42.6)$ & $27(57.4)$ \\
Manual massage & $7(14.9)$ & $40(85.1)$ \\
Hydrocollator & $6(12.8)$ & $41(87.2)$ \\
Kinesiotherapy & $35(74.5)$ & $12(25.5)$ \\
Interferent current & $24(51.1)$ & $23(48.9)$ \\
Diadynamic current & $12(25.5)$ & $35(74.5)$ \\
Electrophoresis & $4(8.5)$ & $43(91.5)$ \\
Galvanic current & $5(10.6)$ & $41(89.4)$ \\
Sonophoresis & $1(2.1)$ & $46(97.9)$ \\
Cryomassage & $3(6.4)$ & $44(93.6)$ \\
\hline
\end{tabular}

TENS: Transcutaneous electrical nerve stimulation 
the highest MMT score at discharge was 5, while the lowest was $2+$.

Out of a total of 47 patients, 30 patients (63.8\%) showed a significant improvement in muscle strength by 1 grade, $1(2.1 \%)$ had a muscle strength improvement by two grades, while 16 (34\%) showed no apparent muscle strength improvements (Figure 1).

Statistical analysis showed that there is a significant difference between the MMT grade at the admission and discharge testing $(p<0.05)$.

Moreover, there was a significant difference observed between the physical MMT rehabilitation and the applied physical therapy modalities $(\mathrm{p}<0.05)$, except in the case of the interferential current (IFC) therapy $(\mathrm{p}=0.132)$. All physical therapy modalities, except IFC, had a significant impact on the results of the MMT rehabilitation (Table 2).

\section{DISCUSSION}

The analysis of demographic data showed the prevalence of female patients with coxarthrosis (76.6\%) over male patients $(23.4 \%)$. Similar results were reported by D'Ambrosia (6) in their research indicating a greater prevalence of coxarthrosis among women. Several other studies also investigated sex distribution in patients with coxarthrosis $(7,8)$.

In contrast to our findings, Tepper and Hochberg found that men had a somewhat higher prevalence of coxarthrosis compared to women $(3.2 \%$ versus
$3.0 \%$ ) (9). They considered that although there was a small difference in the incidence of coxarthrosis between sexes, there were differently represented radiographic patterns of coxarthrosis. Women have more often superomedial forms of coxarthrosis, while men tend to have superolateral (5).

Age is considered the most common predisposing factor for the development of coxarthrosis. This disease commonly occurs in middle-aged people and the elderly aged 60 and above (10).

In their study, Irina et al. found that the incidence of coxarthrosis increases with age (11). Thus, in the age group under 55 years, the incidence rate is $1 \%$; while in the age group over 85 years, it is as high as $10 \%$. The analysis of radiographic images has shown that most people aged 55 and above are affected by osteoarthritis, proving that coxarthrosis is a frequently observed condition in the older population. The patient age representation included the most numerous group aged 70 and above (48.9\%), while the lowest number of patients $(4.3 \%)$ was within the group aged between 41-50.

Finally, the shortest duration of treatment of patients with coxarthrosis was 10-20 days for 11 (23.4\%) patients, while the longest treatment lasted for 31-40 days for 14 (29.8\%) patients (Figure 2).

In our study, we examined the prevalence of individual forms of physical therapy in patients with coxarthrosis. We applied magnet therapy in a total of 17 patients (36.2\%). Some studies have reported

TABLE 2. Correlation of rehabilitation results by manual muscle testing (MMT) and modalities of physical therapy

\begin{tabular}{lllc}
\hline Correlation & T-test & DF & Significant (two-tailed) \\
\hline Magnet therapy and rehabilitation by MMT & -3.685 & 46 & 0.001 \\
Ultrasound and rehabilitation by MMT & -5.636 & 46 & 0.000 \\
TENS and rehabilitation by MMT & -2.595 & 46 & 0.013 \\
Manual massage and rehabilitation by MMT & -5.878 & 46 & 0.000 \\
Hydrocollator and rehabilitation by MMT & -6.970 & 46 & 0.000 \\
Kinesiotherapy and rehabilitation by MMT & -5.596 & 46 & 0.004 \\
Interferent current and rehabilitation by MMT & -1.533 & 46 & 0.132 \\
Diadynamic current and rehabilitation by MMT & -3.774 & 46 & 0.000 \\
Electrophoresis and rehabilitation by MMT & -7.077 & 46 & 0.000 \\
Sonophoresis and rehabilitation by MMT & -8.656 & 46 & 0.000 \\
Cryomassage and rehabilitation by MMT & -7.382 & 46 & 0.000 \\
Galvanic current and rehabilitation by MMT & -6.051 & 46 & 0.000
\end{tabular}

TENS: Transcutaneous electrical nerve stimulation 


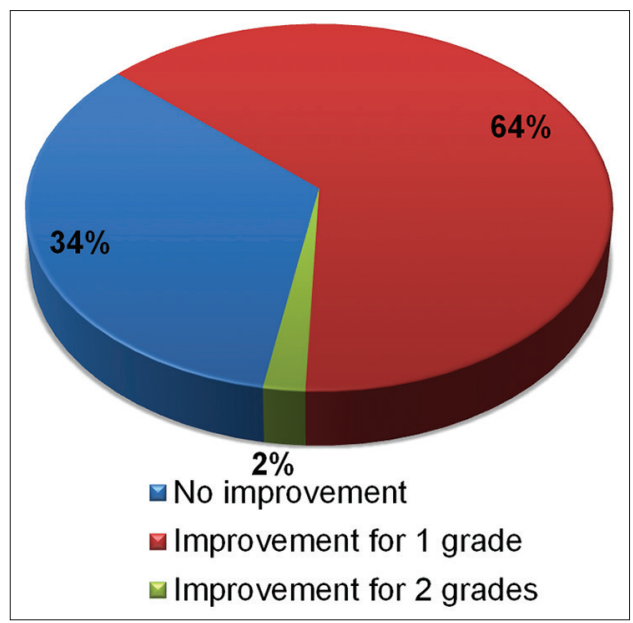

FIGURE 1. Rehabilitation results by manual muscle testing (MMT).

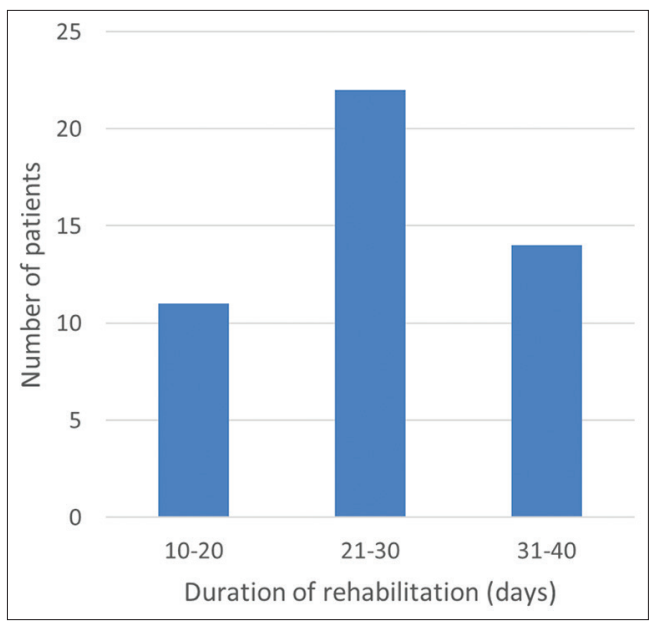

FIGURE 2. Duration of rehabilitation in days.

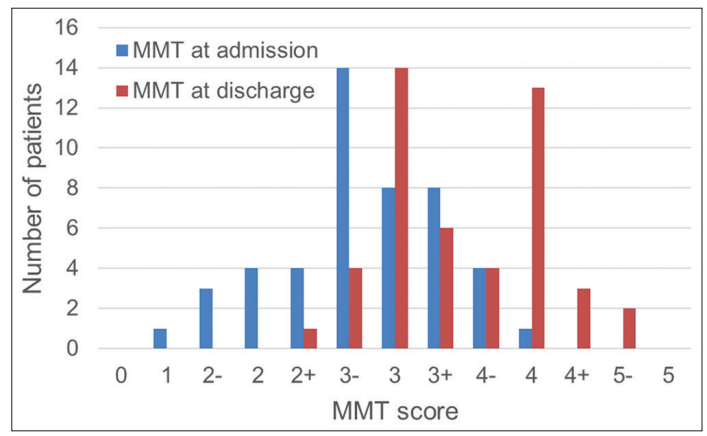

FIGURE 3. Manual muscle testing (MMT) at admission and discharge. beneficial effects of magnet therapy in relieving pain. In a study by Bellamy et al., $29.4 \%$ of patients felt no pain after magnet therapy, $64.7 \%$ felt better, $5.9 \%$ reported no changes, while there were no patients who felt worse than before the treatment (12). Also, ultrasound therapy was applied in 8 patients (17\%). Peter et al. found that ultrasound therapy has no benefit over placebo or short-wave diathermy in the treatment of coxarthrosis (13). Given the lack of evidence on the efficacy of ultrasound therapy, it should be used as supplemental therapy or as a routine option before establishing the final diagnosis. They used TENS in 20 patients $(42.6 \%)$ and the manual massage in 7 patients $(14.9 \%)$ to help reduce pain and improve circulation. Masaaki et al. reported in their study that manual massage has a superior performance over kinesitherapy (14). Many previous studies have shown positive effects of manual massage, and therefore, its application should be considered to provide short-term pain relief and improve the mobility and function of hip joints in patients with coxarthrosis. The hydro collator, as a method of physical therapy, was applied in 6 patients $(12.8 \%)$.

In our study, we applied kinesitherapy in 35 patients, which makes $74.5 \%$ of the total number of patients. Irina et al. reported the importance of kinesiotherapy along with medical treatment and increased mobility in the hip joint and muscle strength as a result of this therapeutic procedure (11).

In their study, Bellamy et al. referred to electrotherapy as not generally recommended in the management of coxarthrosis based on ambiguous evidence or evidence of inefficiency (12).

Based on the values of the MMT ratings at admission and discharge reported in our study, we can conclude that muscle strength has been improved (Figure 3).

\section{CONCLUSION}

All physical modalities (other than IFC) significantly influence muscle strength by the MMT. The MMT values were statistically significantly higher in the discharged patients than the admitted patients, and we can conclude that physical therapy has positively influenced the recovery of muscle strength in patients with coxarthrosis. 


\section{CONFLICT OF INTEREST}

The authors declare no conflict of interest.

\section{REFERENCES}

1. Lane M.D, Nancy E. Osteoarthritis of the hip. New England J Med 2007;20:1413-21.

https://doi.org/10.1056/NEJMcp071112.

2. DiCesare PE, Abramson SB. Pathogenesis of osteoarthritis. In: Harris ED, Budd RC, Firestein GS, Sergent JS, Ruddy S, Sledge CB, editors. Kelley's Textbook of Rheumatology. $7^{\text {th }}$ ed. Philadelphia, PA: Elsevier; 2005. p. 1493-513.

3. Zgoda M, Paczek L, Bartlomiejczyk I, Sieminska J, Chmielewski D, Górecki A. Age-related decrease in the activity of collagenase in the femoral head in patients with hip osteoarthritis. Clin Rheumatol 2007;26:240-1. https://doi.org/10.1007/s10067-006-0365-4.

4. Valdes AM, Spector TD. The clinical relevance of genetic susceptibility to osteoarthritis. Best Pract Res Clin Rheumatol 2010;24(1):3-14. https://doi.org/10.1016/j.berh.2009.08.005.

5. Macovei L, Brujbu I, Murariu RV. Coxarthrosis-disease of multifactorial etiology methods of prevention and treatment. The role of kinesitherapy in coxarthrosis. Rev Med Chir Soc Med Nat lasi 2013;117(2):351-7.

6. D'Ambrosia RD. Epidemiology of osteoarthritis. Orthopedics 2005;28 (2 Suppl):s201-5.

7. O'Connor Ml. Sex differences in osteoarthritis of the hip and knee. J Am
Acad Orthop Surg 2007;15 Suppl 1:S22-5.

https://doi.org/10.5435/00124635-200700001-00007.

8. Puett DW, Griffin MR. Published trials of nonmedicinal and noninvasive therapies for hip and knee osteoarthritis. Ann Intern Med 1994; 121(2):133-40.

https://doi.org/10.7326/0003-4819-121-2-199407150-00010.

9. Tepper S, Hochberg MC. Factors associated with hip osteoarthritis: Data from the first national health and nutrition examination survey (NHANES-I). Am J Epidemiol 1993;137(10):1081-8.

https://doi.org/10.1093/oxfordjournals.aje.a116611.

10. MacDonald CW, Whitman JM, Cleland JA, Smith M, Hoeksma HL. Clinical outcomes following manual physical therapy and exercise for hip osteoarthritis: A case series. J Orthop Sports Phys Ther 2006;36(8):588-99. https://doi.org/10.2519/jospt.2006.2233.

11. Irina A, Hatieganu I. Role of kinesiotherapy in the recovery patients with primary coxarthrosis. Balneo Res J 2013;6:321-50.

12. Bellamy N, Buchanan WW, Goldsmith $\mathrm{CH}$, Campbell J, Stitt LW. Validation study of WOMAC: A health status instrument for measuring clinically important patient relevant outcomes to antirheumatic drug therapy in patients with osteoarthritis of the hip or knee. J Rheumatol 1988;15(12):1833-40.

13. Peter WF, Jansen MJ, Hurkmans EJ, Bloo H, Dekker J, Dilling RG, et al. Physiotherapy in hip and knee osteoarthritis: Development of a practice guideline concerning initial assessment, treatment and evaluation. Acta Reumatol Port 2011;36(3):268-81.

14. Masaaki N, Nagashima T. Long-term follow-up study of conservative therapy for coxarthrosis: Two case studies. Physiotherapy 2001;81:530-5. 